\title{
The relationship between second trimester alpha fetoprotein levels and adverse pregnancy outcome
}

\author{
Rebecca Allen ${ }^{1}$, Shemoon Marleen ${ }^{1}$, Luxmi Velauthar ${ }^{1}$, Kevin Harrington $^{2}$, Joseph Aquilina ${ }^{1}$ \\ ${ }^{1}$ Department of Fetal Medicine, Royal London Hospital, London, UK \\ ${ }^{2}$ Portland Hospital, Great Portland Street, London, UK \\ Email: rebecca_e_allen@yahoo.co.uk
}

Received 29 December 2012; revised 31 January 2013; accepted 9 February 2013

\begin{abstract}
Design: Retrospective analysis. Setting: East London Hospital. Subjects: 724 women who had maternal serum alpha-fetoprotein levels measured between 15 to 19 weeks gestation. Main outcome measure: The main outcome measures were defined as any case of preeclampsia, small-for-gestational age (SGA) birthweight $<5^{\text {th }}$ centile, placental abruption, stillbirth or early neonatal death. Methods: Women with MSAFP $>$ 2.0 Multiples of Median (MoM) were classified as screen positive. Results: 41 (5.7\%) women developed pre-eclampsia. Women with MSAFP > 2.0 Multiples of the Median (MoM) were significantly more likely to develop pre-eclampsia $(p<\mathbf{0 . 0 0 0 0 1})$, deliver a SGA $<5^{\text {th }}$ centile $(\mathrm{p}<0.00001)$, or have a stillbirth/early neonatal death $(p<0.001)$ compared to women with MSAFP $<2.0$ MoM. The odds ratio (OR) for developing pre-eclampsia was $5.2(95 \%$ confidence interval [CI] 2.4 - 11.3), and 8.2 (CI 2.9 - 23.3) for preeclampsia requiring delivery before 37 weeks gestation. Conclusion: Unexplained elevated MSAFP in the second trimester was strongly associated with a subsequent risk of pre-eclampsia especially those requiring preterm delivery, as well as other complications related to uteroplacental insufficiency.
\end{abstract}

Keywords: AFP; Alpha Fetoprotein; Pre-Eclampsia; Small for Gestational Age; Still Birth;

Placental Abruption

\section{INTRODUCTION}

Alpha-fetoprotein is a glycoprotein produced by the fetal liver and gastrointestinal tract. Small amounts of alphafetoprotein cross the placenta and can be measured in the maternal serum. Up to the third month of gestation the majority of fetal production arises from the yolk sac. Increased maternal serum levels of AFP are the consequence of increased amniotic fluid concentration in asso- ciation with fetal defects including exomphalos and open spina bifida; increased transfer from the fetal to maternal circulation as a consequence of placental damage and increased production in the mother from germ cell tumours, hepatocellular carcinoma and metastatic cancer in the liver [1]. It has been used as a marker of open neural tube defects e.g. spina bifida and will detect up to $85 \%$. Most women with unexplained elevated values do not however have a fetus with NTD; in fact only 1 in 15 women with elevated MSAFP has a neural tube defect.

Unexplained mid trimester MSAFP elevations have been associated with an increased risk for low birth weight (LBW), intrauterine growth restriction (IUGR), preterm delivery, placental abruption and preeclampsia. In the first and second trimester evaluation of risk (FASTER) study involving 33,000 women, MSAFP $>2$ MoM was associated with placental abnormalities as well as IUGR (odds ratio 2.7), miscarriage (OR 4.4) and intrauterine fetal death (OR 5.8) [2].

Beta et al. performed a case control study measuring AFP in the $1^{\text {st }}$ trimester which showed a significantly higher median AFP MoM (1.33 vs 0.97, p = 0.006) in those delivering preterm. The estimated detection rate of preterm delivery, at a false positive rate of $10 \%$, from maternal characteristics and obstetric history was $27.5 \%$ which increased to $36 \%$ with the addition of serum AFP [1]. Dehgani-Firouzabadi et al. and Smith et al. also confirmed a raised serum AFP was associated with preterm labour ( $p=0.021$ and $p=0.001$ respectively) $[3,4]$. Waller et al. showed a strong gradient of increasing risk of preterm birth with increasing levels of serum AFP ( $p \leq$ 0.01) [5].

Wald et al. showed that mean birth weight of infants born with unexplained MSAFP elevations > $3.0 \mathrm{MoM}$, was $344 \mathrm{~g}$ less than the birth weight of infants born to women with MSAFP values between 0.75 and 1.49 MoM [6]. Brock et al. (1979) and Burton (1988) also confirmed the association between low birth weight and unexplained elevated MSAFP. Brock et al. reported 
among women who had unexplained elevated MSAFP values greater than $2.3 \mathrm{MoM}, 10.7 \%$ delivered infants weighing less than $2500 \mathrm{~g}$ compared with $4.2 \%$ of women with normal MSAFP values [7]. Burton found that amongst women who had elevated MSAFP values $>2.5$ MoM, 15\% delivered small for gestational age infants compared to only $7.2 \%$ among women with normal MSAFP values [8]. More recent studies have also confirmed these findings [5,9-11]. However, studies by Smith and McPhearson showed no significant increase in IUGR with an elevated AFP [4,12].

There is also an association between elevated MSAFP and fetal demise. Robinson et al. reported that in women with MSAFP values $2.5-2.9 \mathrm{MoM}$, there is a $6 \%$ risk of fetal death, in women with MSAFP values 3.0 - 3.9 MoM , there is a $9 \%$ risk of fetal death and for values $>4.0 \mathrm{MoM}$ the risk of fetal death is $24 \%$ [13]. In a case control study, Walker et al. reported that for women with MSAFP levels > 3.0 MoM, the odds ratio for fetal death was 11:1 (this result being significant). This association was also confirmed by Robinson et al. (1989) who reported a relative risk of 4.7 for neonatal death in cases of unexplained, elevated MSAFP [13]. The risk of stillbirth/early neonatal death appears to increase as the MoM increase. However, the sensitivity and predictive value of using elevated MSAFP for fetal demise or neonatal death is low with sensitivities ranging between $8 \%$ - 30\%. Simpson (1990) concluded that although unexplained elevated MSAFP is significantly associated with increased risk for fetal/neonatal loss, it does not translate into an effective screening test. More recently Krause et al. also found a significant association with an MSAFP MoM > 2.5 and still birth $(\mathrm{p}=0.001)$. However, when adjusted for low birth weight the association was insignificant [10].

Walters et al. reported that $13 \%$ of women with elevated MSAFP developed pre-eclampsia compared to $1 \%$ of the women with normal MSAFP [14]. Williams et al. (1992) compared 201 women with unexplained elevated MSAFP (greater than and including 2.0 MoM) with 211 women with normal MSAFP. A significant association was found between elevated MSAFP and pre-eclampsia, adjusted risk ratio (ARR) being 3.8. Several other studies have also confirmed these finding [2,3,11]. However Kang, Wald and Davidson did not find any significant increase in AFP in those that developed pre-eclampsia compared to controls [15-17].

The null hypothesis for our study is that there is no relationship between second trimester maternal serum alphafetoprotein and the subsequent development of preeclampsia, SGA, stillbirth and neonatal death. If the hypothesis is disproved an initial assessment of its potential value as an early screening test for the condition will be performed.

\section{METHODS}

A retrospective analysis was undertaken of consecutive unselected women who had AFP measured as part of the serum screening program for trisomy 21 in an inner-city teaching hospital. Exclusion criteria were: multiple pregnancies, diabetic pregnancies, hypertension diagnosed before 20 weeks gestation, pregnancies with a prenatal/postnatal diagnosis of a chromosomal or structural abnormality.

All the maternal serum samples were collected between 15 and 19 completed weeks gestation estimated from the last menstrual period or from early ultrasound dates when the menstrual dates differed by more than two standard deviations for the specified gestation. Table 1 summarises the demographic characteristics of the study population. Data collected retrospectively included age, race, previous obstetric history, parity, past medical history, smoking habit, and blood pressure before 20 weeks pregnancy. The main outcome measures were: development of pre-eclampsia. Secondary outcome measures were: gestational age at delivery, gestational hypertension, delivery of a small for gestational age (SGA) baby $<5$ th centile, placental abruption, stillbirth and early neonatal death. The development of gestational hypertension and pre-eclampsia were based on the definitions given by Davy and McGillvray: gestational hypertension was defined as the occurrence in a previously normotensive and non-proteinuric women of 1) a diastolic blood pressure greater than or equal to $90 \mathrm{mmHg}$ on at least two consecutive occasions at least 4 hours apart after the $20^{\text {th }}$ week of gestation or 2) a diastolic blood pressure greater than or equal to $110 \mathrm{mmhg}$ on a single occasion after the $20^{\text {th }}$ week of gestation. Pre-eclampsia was diagnosed when gestational hypertension was associated with significant proteinuria which was defined as $>300 \mathrm{mg}$ proteinuria on 24 hour urinary collection of at least ++ on protein stick-testing on two separate occasions, four

Table 1. Demographics of study population.

\begin{tabular}{cc}
\hline Characteristic & Number $(\%) \mathrm{n}=724$ \\
Caucasian & $383(52.9)$ \\
Afro-Caribbean & $289(39.9)$ \\
Oriental & $43(5.9)$ \\
Nulliparous & $7(0.9)$ \\
Smoker & $354(48.8)$ \\
Previous severe pre-eclampsia & $166(22.9)$ \\
Previous babies $<2500$ g after 37 weeks & $21(2.9)$ \\
Previous premature delivery & $11(1.5)$ \\
\hline
\end{tabular}


hours apart, without evidence of urinary tract infection. "Severe pre-eclampsia" was defined as any case of preeclampsia requiring delivery before 37 completed weeks. "Other" premature deliveries were defined as deliveries before 37 completed weeks in the absence of preeclampsia or gestational hypertension. The diagnosis of placental abruption was made when a clinical diagnosis of antepartum haemorrhage and abdominal pain was associated with a retroplacental clot on examination of the placenta post-delivery. An intrauterine death was defined as stillbirth (SB) if it occurred after 24 completed weeks of gestation, confirmed by ultrasound dating if the menstrual dates were uncertain. A neonatal death (NND) was defined as one occurring within the first week of life. The definition of being SGA at birth was based on being $<5^{\text {th }}$ centile on charts currently in use at our hospital. "Any complication" included all the case of pre-eclampsia or delivery of an SGA $\left(<5^{\text {th }}\right.$ centile) baby or placental abruption, or a pregnancy that resulted in a stillbirth or early neonatal death.

The data was analysed using a Windows based SPSS statistical package version 6.1.3. Statistical significance was assessed using Chi-squared test or Fisher's Exact test where cell counts were small. The Wilcoxon rank sum test was used to compare the distribution of alpha fetoprotein values in women with known outcome and those lost to follow-up. Statistical significance was set at $\mathrm{p}<0.05$ level.

\section{RESULTS}

Tables 2 and 3 summarise the results. 41 women developed pre-eclampsia $(5.7 \%)$ of which $17(2.3 \%)$ required delivery before 37 weeks gestation. 80 women delivered SGA fetuses less than the $5^{\text {th }}$ centile $(11 \%) .18$ women $(2.5 \%)$ delivered stillbirths or suffered from early neonatal deaths. 5 women $(0.7 \%)$ had an abruption. 50 women $(6.9 \%)$ had MSAFP greater than 2.0 MoM. These women were defined as screen positive and the rest were defined as screen negative. $20 \%$ of screen positive women developed pre-eclampsia compared to $4.6 \%$ of the screen negative women.

\subsection{Small for Gestational Age}

The Pearson Chi-Squared test gave a result of $\mathrm{p}=0.0001$, this is significant. Odds ratio (confidence interval) is 7.6 (4.1 to 14.1). The sensitivity, false positive rate, specificity, positive predictive value, negative predictive value calculated are, respectively, 26\%, 3.4\%, 96\%, 42\% and $91 \%$.

\subsection{Abruption}

The Pearson Chi-Squared test gave a result of $\mathrm{p}=0.54$, this is not significant. Odds ratio is $0.9(0.98-0.99)$. The sensitivity, false positive rate, specificity, positive predictive value, negative predictive value calculated are, respectively, $0 \%, 93 \%, 7.0 \%, 0 \%, 99.3 \%$.

\subsection{Stillbirth/Neonatal Death}

The Pearson Chi-Squared test gave a result of $\mathrm{p}=0.0001$, this is significant. Odds ratio is 7.534 (2.699 to 21.027). The sensitivity, false positive rate, specificity, positive predictive value and negative predictive value calculated are, respectively, $33.3 \%, 6.2 \%, 93.8 \%, 12.0 \%$ and $98 \%$.

\section{DISCUSSION}

AFP is initially formed by the yolk sac and then the fetal liver with a smaller contribution from the gastrointestinal tract. Maternal serum AFP rises from weeks 10 to 32 and then declines. Fetal to maternal transfer of AFP is thought to be via two routes, transplacental and transmembranous. In a small morphometric study of placentas associated with raised MSAFP, a normal concentration of amniotic fluid AFP, and a normally formed fetus, the mean total placental volume, volume of parenchyma and villous

Table 2. Summary of results for each outcome.

\begin{tabular}{|c|c|c|c|c|c|}
\hline Outcome & $\mathrm{n}($ total $)=724(\%)$ & $\leq 2.0 \mathrm{MoM} \mathrm{n}=674(\%)$ & $>2 \mathrm{MoMn}=50(\%)$ & $\mathrm{p}$ & OR $(95 \% \mathrm{CI})$ \\
\hline Pre-eclampsia & $41(5.7)$ & $31(4.6)$ & $10(24.4)$ & $<0.0001$ & $5.2(2.4-11.3)$ \\
\hline Pre-eclampsia $<37$ weeks & $17(2.3)$ & $11(1.6)$ & $6(12)$ & $<0.0001$ & $8.2(2.9-23.3)$ \\
\hline $\mathrm{PIH}$ & $20(2.8)$ & $16(2.4)$ & $4(8)$ & 0.02 & $3.6(1.1-11.3)$ \\
\hline $\mathrm{SGA}<5^{\text {th }}$ centile & $80(11)$ & $59(8.7)$ & $21(42)$ & $<0.0001$ & $7.6(4.1-14.1)$ \\
\hline Premature delivery & $56(7.7)$ & $47(7)$ & $9(18)$ & 0.005 & $2.9(1.3-6.4)$ \\
\hline Abruption & $5(0.7)$ & $5(0.8)$ & - & 0.54 & $0.9(0.98-0.99)$ \\
\hline Stillbirth/NND & $18(2.5)$ & $12(1.8)$ & $6(12)$ & $<0.0001$ & $7.5(2.7-21)$ \\
\hline All complications & $107(14.8)$ & $81(12)$ & $26(52)$ & $<0.0001$ & $7.9(4.3-14.4)$ \\
\hline
\end{tabular}


surface area were increased, there were more areas of infarction and a lower fetal-placental weight ratio compared with the control group [18]. Boyd et al. propose that the reason $2^{\text {nd }}$ trimester AFP may be raised in combination with a normally formed fetus can be attributed mainly to changes in the placenta which has the property of responding to an adverse environment by increasing its surface of exchange. Brazerol et al. reported that the explanation for the association between elevated maternal serum alpha fetoprotein and adverse pregnancy outcome is not clear, but is probably a marker of placental dysfunction, including partial placental abruption, fetomaternal bleeding and abnormal implantation [19].

Our study shows there is a highly significant association between elevated maternal serum alpha-fetoprotein levels (in this study the "normal" cut off defined as 2.0 $\mathrm{MoM}$ ) and each of the adverse pregnancy outcomes of pre-eclampsia, baby small for gestational age and stillbirth/neonatal death. In this study, it has been shown that there are increased odds of each of the adverse pregnancy outcomes if a pregnant mother has a MSAFP $>2.0$ MoM opposed to a pregnant mother who has a MSAFP $<$ 2.0 MoM.

The question is this a good enough screening tool for pre-eclampsia? A clinically useful screening test must have high sensitivity, a clinically relevant positive predictive value and acceptable false-positive rate (high specificity). If this was used as a screening test, and we looked at all the women who had a result of second trimester MSAFP level higher than 2.0 MoM, for every one pregnant woman diagnosed as affected there will be 4 who are not affected, the sensitivity is only $24 \%$. Although it might not satisfy the criteria of a very effective screening test because of the low sensitivity, for screen positive women it does not do any harm to check the blood pressure and urinalysis more frequently in the second half of pregnancy, as these women are at a sig- nificantly increased risk of pre-eclampsia. Audibert et al. found that an elevated MSAFP level in combination with the presence of uterine artery notching on Doppler ultrasound improved the positive predictive value to $21 \%$ from $3.9 \%$ for pre-eclampsia and $43 \%$ from $12.5 \%$ for IUGR [11].

For small for gestational age less than $5^{\text {th }}$ centile a positive predictive value of $42 \%$ is more than acceptable but the sensitivity was only $26 \%$. So although the sensitivity is low, women with MSAFP greater than $2.0 \mathrm{MoM}$ could be offered increased antenatal surveillance in the form of ultrasound scans for growth.

In terms of stillbirth/neonatal death even if the OR is as low as 2.699 , looking at all the pregnant women who had a second trimester MSAFP result of more than 2.0 MoM, for every 3 pregnant women who eventually proceeds to have a stillborn baby or experience a neonatal death, there will be 22 women who experience neither. Table 3 shows 18 pregnant women had a stillborn infant or a neonatal death. This data implies a prevalence of still-birth/neonatal death of approximately $2.5 \%$. This is quite rare. With a false positive rate of $6.2 \%$, approximately $6 \%$ of the total of women screened will give a positive result, but will not have a stillborn infant or neonatal death.

With regards to abruption, and a cut off point of 2.0 $\mathrm{MoM}$, the sensitivity is $0 \%$, the false positive rate is $7 \%$ and the positive predictive value is $0 \%$. The number of data analysed is small, so in this case, it seems that unreliable figures are given.

In view of our studies results, which are in consensus with a number of previous studies, we would recommend offering women with a raised MSAFP, aspirin $75 \mathrm{mg}$ daily and a uterine artery Doppler scan. If the mean PI is $>1.6$ they would be categorised as screen positive and offered $150 \mathrm{mg}$ aspirin daily and serial growth scans in the $3^{\text {rd }}$ trimester.

Table 3. Summary sensitivities, specificities, false positive rates, positive and negative predictive values.

\begin{tabular}{|c|c|c|c|c|c|}
\hline Outcome & Sensitivity & Specificity & False positive rate & PPV & NPV \\
\hline Pre-eclampsia & 24 & 94 & 5.9 & 20 & 95 \\
\hline Pre-eclampsia $<37$ weeks & 35 & 94 & 6 & 12 & 98 \\
\hline $\mathrm{PIH}$ & 20 & 94 & 6 & 8 & 98 \\
\hline $\mathrm{SGA}<5^{\text {th }}$ centile & 26 & 96 & 3.4 & 42 & 91 \\
\hline Premature Delivery & 16 & 94 & 6 & 18 & 93 \\
\hline Placental abruption & 0 & 7 & 93 & 0 & 99.3 \\
\hline Stillbirth/NND & 33 & 94 & 6.2 & 12 & 98 \\
\hline All Complications & 24 & 96 & 4 & 52 & 88 \\
\hline
\end{tabular}




\section{CONCLUSION}

Pregnant women with maternal serum alpha fetoprotein $>2.0 \mathrm{MoM}$ (measured between 15 to 19 weeks) do have increased odds of an outcome of pre-eclampsia, a baby small for gestational age and stillbirth/neonatal death. The results show that for pre-eclampsia and stillbirth/ neonatal death, maternal serum alpha fetoprotein with a cut off point of 2.0 MoM as normal would not be a good screening test. But, a pregnant woman, whose maternal serum alpha fetoprotein level $>2.0 \mathrm{MoM}$ (measured at 15 to 19 weeks) has significantly increased odds of an outcome of pre-eclampsia and stillbirth/neonatal death than a woman with maternal serum alpha-fetoprotein levels $<2.0 \mathrm{MoM}$. The results are not only statistically, but also clinically significant. It would therefore be worthwhile testing pregnant women at 15 to 19 weeks for their maternal serum alpha fetoprotein levels. For the outcome small for gestational age, the positive predictive value is $60 \%$, so maternal serum alpha fetoprotein $(>2.0 \mathrm{MoM})$ would be a useful screening test, although the detection rate is low.

\section{REFERENCES}

[1] Beta, J.B.F., Rodriguez, J., Akolekar, R. and Nicolaides, K. (2011) Maternal serum alphafetoprotein at $11-13$ weeks' gestation in spontaneous early preterm delivery. Fetal Diagnosis and Therapy, 30, 88-93. doi:10.1159/000324352

[2] Dugoff, L.H.J., Nalone, F., Vidaver, J., Sullivan, L. and Canick, J. (2005) Quad screen as a predictor of adverse pregnancy outcome. Obstetrics and Gynecology, 106, 260-267. doi:10.1097/01.AOG.0000172419.37410.eb

[3] Dehghani-Firouzabadi, R.T.N., Ghasemi, N. and Tahmasbi, Z. (2010) The association between second-trimester maternal serum alpha-fetoprotein in $14-22$ weeks and adverse pregnancy outcome. Acta Medica Iranica, 48, 234-238

[4] Smith, G., Shah, I., Crossley, J., Aitken, D., Pell, J., Nelson, S. et al. (2006) Pregnancy associated plasma protein A and Aplpha-fetoprotein and prediction of adverse perinatal outcome. Obstetrics and Gynecology, 107, 161-166. doi:10.1097/01.AOG.0000191302.79560.d8

[5] Waller, K.L.L., Cunningham, G., Feuchtbaum, L. and Hook, E. (1996) The association between maternal serum alpha-fetoprotein and preterm birth, small for gestational age infants, preeclampsia and placental complications. Obstetrics and Gynecology, 88, 816-822.

[6] Wald, N.J., Cuckle, H. and Brock, J.H.L. (1977) Maternal serum-alpha-fetoprotein measurement and low birth weight. Lancet, 1, 1323-1332.

[7] Brock, D.J., Barron, L. and Watt, M. (1979) The relation between maternal plasma alpha-fetoprotein and birth weight in twins. British Journal of Obstetrics and Gynae- cology, 86, 710-712.

doi:10.1111/j.1471-0528.1979.tb11271.x

[8] Burton, B.K. (1988) Outcome of pregnancy in patients with unexplained elevated or low levels of. Obstetrics \& Gynecology, 72, 709-713

[9] Morssink, L. P., de Wolf, B.T., Kornman, L.H., Beekhuis, J.R., van der Hall, T.P.J. and Mantingh, A. (1996) The relation between serum markers in the second trimester and placental pathology. A study on extremely small for gestational age fetuses. British Journal of Obstetrics and Gynaecology, 103, 779-783.

[10] Krause, T., Christens, P., Wohlfahrt, J., Lei, U., Westergaard, T., Norgaard-Pedersen, B., et al. (2001) Second trimester maternal serum alpha-fetoprotein and risk of adverse pregnancy outcome. Obstetrics and Gynecology, 97, 277-282.

[11] Audibert, F.B.Y., Benhatter, C., Champagne, C. and Frydman, R. (2005) Prediction of preeclampsia or intrauterine growth restriction by second trimester serum screening and uterine doppler velocimetry. Fetal Diagnosis and Therapy, 20, 48-53.

[12] Mc Pherson, E., Thomas, G., Manlick, C., Zaleski, C., Reynolds, K., Rasmussen, K. et al. (2011) Extreme values of maternal serum analytes in second trimester screening. Looking beyond trisomy and NTD's. Journal of Genetic Counselling, 20, 396-403.

[13] Robinson, L., Grau, P. and Crandall, B.F. (1989) Pregnancy outcomes after increasing maternal serum alphafetoprotein levels. Obstetrics \& Gynecology, 74, 17-20.

[14] Walters, B.N., Lao, T., Smith, V., et al. (1985) Alphafetoprotein elevation and proteinuric pre-eclampsia. British Journal of Obstetrics and Gynaecology, 92, 341-344.

[15] Kang, J., Farina, A., Park, J., Kim, S., Kim, J., Rizzo, N. et al. (2008) Down Syndrome biochemical markers and screening for preeclampsia at first and second trimester: Correlation with the week of onset and severity. Prenatal Diagnosis, 28, 704-709.

[16] Wald, N. and Morris, J. (2001) Multiple marker second trimester serum screening for pre eclampsia. Journal of Medical Screening, 8, 65-68.

[17] Davidson, E., Riley, S., Roberts, S., Shearing, C., Groome, N. and Martin, C. (2003) Maternal serum activin, inhibin, human chorionic gonadotrophin and alpha-fetopprotein as second trimester predictors of preeclampsia. British Journal of Obstetrics and Gynaecology, 110, 543552.

[18] Boyd, P. and Keeling, J. (1986) Raised maternal serum alpha- fetoprotein in the absence of fetal abnormality-placental findings. A quantitative morphometric study. Prenatal Diagnosis, 6, 369-373.

[19] Brazerol, W.F., Grover, S. and Donnenfield, A.E. (1994) Unexplained elevated maternal serum alpha-fetoprotein levels and perinatal outcome in an urban clinic population. American Journal of Obstetrics and Gynecology, 171, 1030-1035. doi:10.1016/S0029-7844(00)01109-1 